\title{
Impact of Slip Cycles on the Operation Modes and Efficiency of Molecular Motors
}

\author{
Steffen Liepelt • Reinhard Lipowsky
}

Published online: 26 August 2010

(C) The Author(s) 2010. This article is published with open access at Springerlink.com

\begin{abstract}
Kinesin is a motor molecule that moves processively on microtubule tracks and is involved in active intracellular transport processes. For small loads, it is powered by the hydrolysis of one ATP molecule per step. Here we extent our previously introduced network theory in order to study the possibility of two different mechanical stepping transitions and the general behavior of the motor's efficiency. Our theory shows explicitly how chemical and mechanical slip cycles emerge that weaken the coupling between ATP hydrolysis and mechanical stepping. Near chemomechanical equilibrium, the motor efficiency $\eta$ may vary between $\eta=1$ for tight coupling and $\eta=0$ for loose coupling, depending on the relevance of the slip cycles. Far from chemomechanical equilibrium, on the other hand, the motor efficiency is found to decay as $1 / \Delta \mu$ with increasing $\Delta \mu$ irrespective of the presence of slip cycles, where $\Delta \mu$ represents the reaction free enthalpy or chemical potential difference per ATP hydrolysis.
\end{abstract}

Keywords Chemomechanical coupling · Operation modes $\cdot$ Motor efficiency

\section{Introduction}

Molecular motors convert chemical energy into mechanical work. More precisely, these motors act as enzymes that increase the rate of certain chemical reactions such as ATP hydrolysis, undergo a specific sequence of conformational transitions, and move in a directed manner even though they experience relatively strong thermal noise. Different theoretical approaches have been used to describe this interplay of chemistry and mechanics. Roughly speaking, one may distinguish two different types of motor descriptions starting from 'mechanical ratchets' and 'chemical networks', respectively.

\footnotetext{
S. Liepelt $(\bowtie) \cdot R$. Lipowsky

Theory and Bio-Systems, Max-Planck-Institute of Colloids and Interfaces, Science Park Golm, Potsdam 14424, Germany

e-mail: liepelt@mpikg.mpg.de

R. Lipowsky

e-mail: lipowsky@mpikg.mpg.de
} 
The ratchet approach focuses on the motor's mechanical movement and views the motor as a small particle that is subject to an asymmetric force potential. Presumably, the first theoretical description of this kind was used by Smoluchowski [1], who showed that an asymmetric force potential cannot lead to directed motion of the particle as long as this particle is in equilibrium with its surroundings. In order to obtain directed motion, the motor particle has to be coupled to a switching mechanism that effectively leads to two or several motor states. The switching enables the particle to rectify the thermal fluctuations. Such a mechanism was proposed in the classical sliding model of Huxley [2] for the interaction of myosin and actin. For the stepping motor kinesin, a similar approach was introduced by Peskin and Oster [3]. In fact, a rather large number of ratchet models has been studied theoretically as reviewed in [4] and [5].

The network approach, on the other hand, starts from the enzymatic activity of the motor which implies a certain network of discrete chemical states and chemical transitions between these states. These chemical transitions are uniquely and unambiguously defined by the underlying chemical reaction. The network approach has its roots in transition state $[6,7]$ and reaction rate [8] theories as well as in enzyme kinetics $[9,10]$. We have recently extended this latter approach by adding mechanical transitions to these chemical networks which leads to rather concise theoretical representations for the chemomechanical coupling of the motors [11-13].

Within the ratchet models, the spatial displacement of the motor is described by a continuous coordinate. If one discretizes this coordinate, one obtains discrete, sequential stochastic models as studied in Refs. [14-16]. These latter models correspond to networks with a single motor cycle. As explained elsewhere, [12] such uni-cycle models are inadequate to describe stepping motors such as kinesin that are able to undergo processive backstepping in the presence of a resisting load and in the absence of the hydrolysis products.

One important distinction between the ratchet and the network approach arises from the different time scales involved in the chemical and mechanical transitions. The ratchet approach assumes that the mechanical motion is slow compared to the chemical kinetics. This is intuitively appealing since the mechanical stepping involves tens of nanometers whereas the chemical reactions are confined to the nucleotide binding pocket with a size of the order of $1.5 \mathrm{~nm}$. However, recent experiments have shown that the separation of time scales does not follow the separation of length scales. Indeed, in the absence of load, the mechanical transitions of stepping motors are completed within microseconds [17] whereas the hydrolysis reactions take many milliseconds. Such a counterintuitive separation of time scales can be easily incorporated in the network approach, in which the chemical and mechanical transitions are treated on equal footing.

Another advantage of the network approach is that it allows us to classify the different types of motor cycles [13]. Furthermore, it is straightforward to assign fluxes to each cycle of a network representation, which makes the discussion of the motor dynamics particularly transparent $[10-13,18]$.

In general a network of conformational states of a dimeric motor, such as kinesin, includes a large number of cycles, see Fig. 1(a). Of course, not all of these cycles are equally relevant for the observed motor dynamics. An interesting and important theoretical challenge is to identify the most relevant cycles, to explore their competitive dynamics, and to relate this dynamics to observable phenomena. In order to achieve these objectives, it is very useful to classify the different motor cycles according to their energy transduction ability. Chemomechanical cycles couple the consumption of substrate molecules such as ATP to mechanical displacements. On the other hand, chemical or enzymatic slip cycles correspond to substrate consumption not coupled to mechanical transitions, whereas mechanical slip cycles describe motor displacements that are not coupled to any chemical turnover. Obviously, 

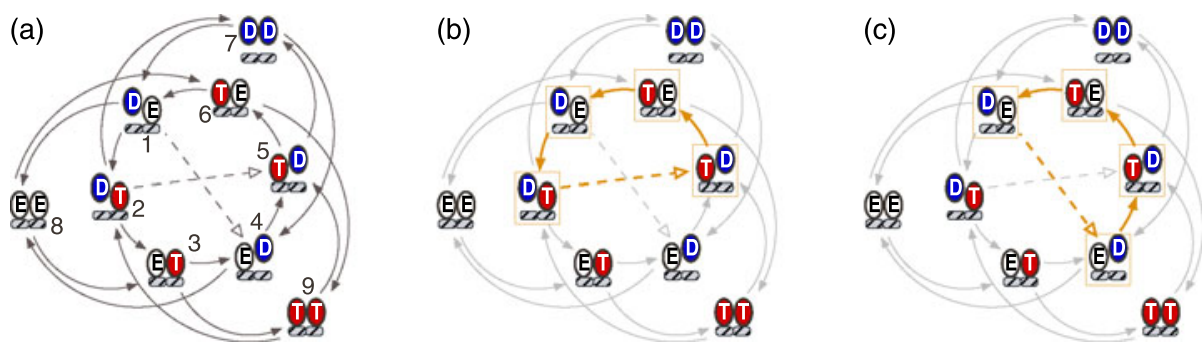

Fig. 1 Chemomechanical network of kinesin: (a) Nucleotide state space of kinesin with two possible mechanical transitions (broken lines) as described in [29]; (b) Forward chemomechanical cycle (orange) with mechanical stepping from motor state DT to motor state TD induced by ATP binding, as in e.g. [23-25]; and (c) Forward chemomechanical cycle (orange) with mechanical stepping from motor state DE to motor state ED induced by $\mathrm{P}$ release, as in e.g. [26-28]

to obtain an efficient and tightly coupled motor that does not waste substrate molecules, one would like to reduce the role of slip cycles. However, for kinesin [11] as well as for myosin under different external conditions, experimental observations imply that different chemomechanical cycles are used. The network of the different chemomechanical cycles involves, in general, enzymatic and/or mechanical slip cycles as well.

In this article, we show how the operation mode boundaries and the motor efficiency are affected by the emergence of chemical and mechanical slip cycles. In the regime close to equilibrium, we are able to obtain explicit relations between the fluxes on slip cycles and the chemomechanical coupling parameters, that determine the operation mode boundaries and the motor efficiency. Furthermore, we also show that, far away from equilibrium, the motor efficiency may exhibit a maximum as a function of the reaction free enthalpy $\Delta \mu$, which arises from the presence of slip cycles, but always decays as $1 / \Delta \mu$ in the limit of large $\Delta \mu$ irrespective of the presence of such cycles.

\subsection{Motor Properties of Kinesin: A Brief Review}

In this study, conventional kinesin serves as a paradigmatic example to analyze the different operation modes for the energy transduction by molecular motors.

Kinesin is a dimeric motor with two identical motor heads that catalyze the hydrolysis of ATP and, at the same time, generate the directional movement along microtubules. At small load forces, the kinesin motor exhibits tight chemomechanical coupling, i.e. it hydrolyzes one ATP molecule per mechanical step [19]. To achieve the remarkably high processivity of about 100 steps the two identical heads are coordinated in such a way that at least one of them is always bound to the microtubule [20]. As a result, the motor walks hand-over-hand with its two heads alternating as leading and trailing heads [21]. Kinesin moves via fast stepping events of $8 \mathrm{~nm}$ within less than $15 \mu \mathrm{s}$, interrupted by comparably long dwell times that are on average longer than $10 \mathrm{~ms}$ [17]. These dwell times are limited by the catalysis of ATP and include processes of ATP binding, ATP cleavage with rapid subsequent P release, and ADP release. The corresponding chemical state space of the dimeric kinesin molecule involves nine nucleotide states and is depicted in Fig. 1(a). It is generally agreed that kinesin under low load is governed by a dominant forward cycle but the precise nature of this cycle, in particular the incorporation of the step transition, is not known.

Since both the empty (rigor) head and the ATP head bind strongly to microtubules compared to the ADP head [22], one identifies two candidates for the dominant fast stepping 
transition during processive motion. The weakly bound ADP head might move in front of a strongly bound ATP head corresponding to a transition from the DT state to the TD state, as in Fig. 1(b). Alternatively, the ADP head might move in front of a strongly bound empty head. Then a motor step corresponds to the transition from state DE to state ED, see Fig. 1(c). Inspection of Fig. 1 shows that the two proposals in Fig. 1(b) and Fig. 1(c) can also be distinguished by the temporal ordering of ATP binding and mechanical stepping: in Fig. 1(b), ATP is bound before mechanical stepping whereas it is bound after stepping in Fig. 1(c). In the literature, one finds both proposals corresponding to Fig. 1(b) and (c) for the dominant chemomechanical cycle in the small load regime. Motor cycles that involve stepping triggered by ATP binding on the leading head as in Fig. 1(b) have been proposed in Refs. [23-25]. On the other hand, stepping triggered by the release of phosphate on the trailing head have been discussed in [26-28].

We have previously introduced a network representation for the processively moving kinesin motor $[12,29]$ to establish a thermodynamically consistent description that incorporates the experimental observation of processive backward stepping for super-stall loads $[17,30]$. Since backward stepping of kinesin was faster for larger ATP concentrations and furthermore appeared under conditions of negligible ADP and P concentrations, it could not be explained by simply reversing the dominant chemomechanical cycle for small load forces. Furthermore, the inhibition of the kinesin motor velocity by ADP and P has been shown to be competitive with ATP binding in both cases, [26]. This observation cannot be reproduced by single cycle models [15] that have a well defined sequence of P release, ADP release and ATP binding. All of these experimental observations imply that one has to consider more than one chemomechanical motor cycle, and that the dynamics of kinesin is governed by the interplay and competition of several motor cycles [12, 29, 31].

In our previous analysis of kinesin's motor cycles, we focused on the reduced network as shown in Fig. 2, where we introduce our short-hand notation for the motor states: the states are labeled by index $i$ with $i=1, \ldots, 7$. The chemical or mechanical transitions from state $i$ to state $j$ will be denoted by $|i j\rangle$, whereas an edge, representing both the forward transition $|i j\rangle$ and the backward transition $|j i\rangle$, will be denoted by $\langle i j\rangle$. We also argued in $[12,29]$, that mechanical stepping from DE to ED is unlikely since this transition competes with the ATP binding transition from DE to DT and, thus, should lead to a motor velocity that decreases with increasing ATP concentration. Since such a decrease of the velocity is not observed experimentally, we focused on the mechanical transition $|25\rangle$ from DT to TD. As a result, we obtained rather good agreement with several sets of data for the motor velocity as a function of load force $F$, substrate concentration [ATP] and product concentrations [ADP] and [P], as obtained in single molecule experiments. These latter experiments have been performed far from chemical equilibrium and over a limited range of load forces. Thus, in this experimentally explored regime, one may discard the mechanical transition $|14\rangle$ from motor state DE to motor state ED.

However, for large assisting loads the stepping transition $|14\rangle$ might become more important. Indeed, in motor state 1 , the trailing $\mathrm{D}$ head is only weakly bound and, thus, will be moved forward by a sufficiently strong assisting load. Likewise, if the motor experiences a sufficiently large resisting load, it is likely to undergo the reverse transition $|41\rangle$ from state ED to state DE as recently pointed out in [31]. In the latter study it has been argued, that backsteps from TD to DT are less likely than backsteps from ED to DE, because the trailing $\mathrm{T}$ head in the TD state may have a docked neck linker, which has to unzip in order to allow for a backstep. At present, the experimental data for the high load regimes are rather limited and do not allow to decide between the different mechanical transitions in a definite manner. 

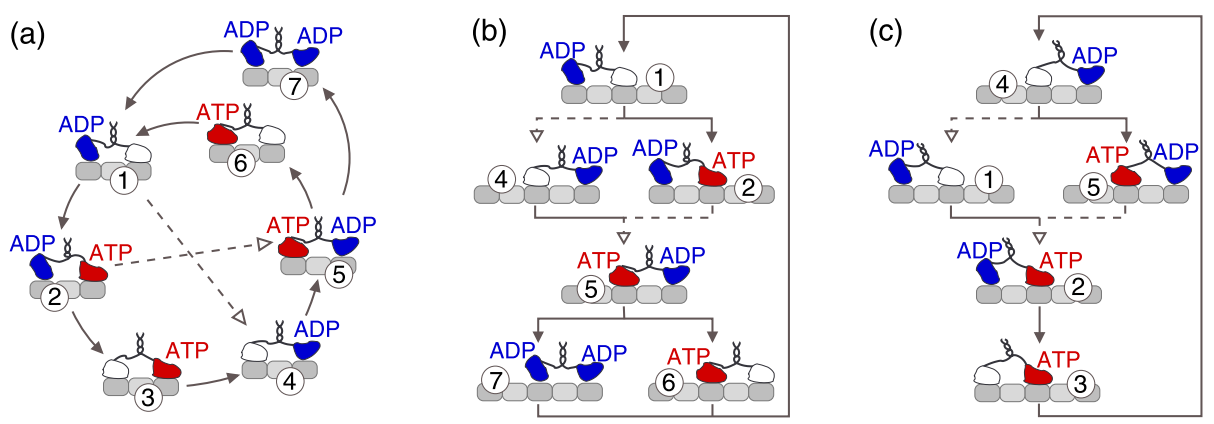

Fig. 2 (a) Reduced network representation for the processive walk of kinesin corresponding to a subnetwork of the complete description in Fig. 1 . The network consists of seven motor states $i=1,2, \ldots, 7$ as indicated by the encircled numbers. (b) Chemomechanical forward stepping cycles. Dashed arrows indicate mechanical transitions towards the plus end of the microtubule. (c) Chemomechanical backward stepping cycles. Dashed arrows indicate mechanical transitions towards the minus end of the microtubule

Since we will study the properties of our kinesin network representation for arbitrary external conditions, including the regime close to chemical equilibrium, we incorporate both mechanical steps $\langle 14\rangle$ and $\langle 25\rangle$ in order to keep a certain level of generality.

\subsection{Cycle Representation}

It is instructive to discuss the motor dynamics of the kinetic network representation in terms of cycles and directed cycles. A cycle corresponds to a set of network states that can be connected by a closed path for which all vertices and edges occur only once. A directed cycle or dicycle is then a sequence of states in one of the two possible directions of the corresponding cycle.

The chemomechanical network shown in Fig. 2 contains two mechanical transitions corresponding to the edges $\langle 25\rangle$ and $\langle 14\rangle$. If we included only one mechanical transition as described previously in [29], the reduced network would have six different cycles. The addition of the second mechanical transition involves only two additional transition rates but increases the number of cycles from six to 12. These twelve cycles are listed in Appendix A. For each dicycle $\mathcal{C}_{v}^{+}$, we denote the numbers of forward and backward mechanical transitions contained in this dicycle by $m_{\mathrm{f}}\left(\mathcal{C}_{v}^{+}\right)$and $m_{\mathrm{b}}\left(\mathcal{C}_{v}^{+}\right)$, respectively. Likewise, we denote the numbers of ATP hydrolysis and synthesis transitions within this dicycle by $n_{\mathrm{h}}\left(\mathcal{C}_{v}^{+}\right)$and $n_{\mathrm{s}}\left(\mathcal{C}_{v}^{+}\right)$. As shown in the following, the quantities that enter the physically relevant fluxes are the differences

$$
m_{v}^{+} \equiv m_{\mathrm{f}}\left(\mathcal{C}_{v}^{+}\right)-m_{\mathrm{b}}\left(\mathcal{C}_{v}^{+}\right)
$$

and

$$
n_{v}^{+} \equiv n_{\mathrm{h}}\left(\mathcal{C}_{v}^{+}\right)-n_{\mathrm{s}}\left(\mathcal{C}_{v}^{+}\right)
$$

For each motor cycle, the average heat $\bar{Q} \equiv Q /\left(k_{B} T\right)$ that is released to the heat bath during cycle completion is given by $[11,32]$

$$
\bar{Q}\left(\mathcal{C}_{v}^{+}\right)=n_{v}^{+} \Delta \bar{\mu}-m_{v}^{+} \bar{F},
$$


where

$$
\Delta \bar{\mu}=\log \left(\frac{K^{\mathrm{eq}}[\mathrm{ATP}]}{[\mathrm{ADP}][\mathrm{P}]}\right)
$$

is the chemical potential difference of ATP hydrolysis in units of $k_{B} T$, and

$$
\bar{F}=\ell F /\left(k_{B} T\right)
$$

is the work per mechanical step of length $\ell$ performed by the motor molecule against the resisting load $F$ in units of $k_{B} T$. We use the convention that resisting and assisting forces correspond to $F>0$ and $F<0$, respectively.

The different catalytic cycles of a kinetic network are characterized as (i) chemomechanical forward stepping cycles $\mathcal{F}$ with $n_{v}^{+}>0$ and $m_{v}^{+}>0$, chemomechanical backward stepping cycles $\mathcal{B}$ with $n_{v}^{+}>0$ and $m_{v}^{+}<0$, enzymatic slip cycles $\mathcal{E}$ with $n_{v}^{+}>0$ and $m_{v}^{+}=0$, and mechanical slip cycles $\mathcal{M}$ with $n_{v}^{+}=0$ and $m_{v}^{+}>0$, Appendix A.

Whereas thermodynamics determines the heat dissipation of cycle completion, kinetics determines the rate of cycle completion. The number of cycle completions per second is given by

$$
J\left(\mathcal{C}_{v}^{+}\right)=\left(\prod^{\mathcal{C}_{v}^{+}} \omega_{i j}\right) \frac{\Upsilon\left(\mathcal{C}_{v}\right)}{\Omega}>0,
$$

which is a function of the individual transition rates $\omega_{i j}$ between the states of the kinetic network representation. The statistical weight $\Upsilon\left(\mathcal{C}_{v}\right) / \Omega$ describes the flux onto the cycle $\mathcal{C}_{v}$ from the rest of the kinetic network [18].

A direct link between thermodynamics and kinetics is provided by the ratio of the number of cycle completions in one direction and the number of cycle completions in the reverse direction, which is related to the dissipated heat $\bar{Q}$ for the corresponding cycle and given by [13]

$$
\frac{J\left(\mathcal{C}_{v}^{+}\right)}{J\left(\mathcal{C}_{v}^{-}\right)}=\prod^{\mathcal{C}_{v}^{+}} \frac{\omega_{i j}}{\omega_{j i}}=e^{\bar{Q}\left(\mathcal{C}_{v}^{+}\right)} .
$$

The excess flux $\Delta J\left(\mathcal{C}_{v}^{+}\right)$of a directed cycle $\mathcal{C}_{v}^{+}$in a kinetic network is defined by the difference between $J\left(\mathcal{C}_{v}^{+}\right)$and $J\left(\mathcal{C}_{v}^{-}\right)$. Using the relation (7), the reduction of the excess flux on a cycle by the reverse process can also be expressed by a factor that depends on the dissipated heat $Q\left(\mathcal{C}_{v}^{+}\right)$of the cyclic forward process, which leads to [29]

$$
\Delta J\left(\mathcal{C}_{v}^{+}\right)=J\left(\mathcal{C}_{v}^{+}\right)-J\left(\mathcal{C}_{v}^{-}\right)=J\left(\mathcal{C}_{v}^{+}\right)\left(1-e^{-\bar{Q}\left(\mathcal{C}_{v}^{+}\right)}\right)
$$

Equation (8) quantitatively connects the irreversibility of a catalytic process and its heat dissipation. The heat release and the flux on a catalytic cycle vanish simultaneously, i.e. $\Delta J\left(\mathcal{C}_{v}^{+}\right)=0$ if and only if $\bar{Q}\left(\mathcal{C}_{v}^{+}\right)=0$. If the latter is true for all cycles of the kinetic network, the motor is in equilibrium. In a non-equilibrium situation, the flux on an isothermal cycle will always proceed in the direction of heat release. Indeed the statement "An isothermal cycle cannot convert heat into work" represents one of the many formulations of the second law of thermodynamics [33].

With a positive $\bar{Q}\left(\mathcal{C}_{v}^{+}\right)$in (8), also the flux $\Delta J\left(\mathcal{C}_{v}^{+}\right)$is positive, which implies that the system evolves on the cycle $\mathcal{C}_{v}$ in the plus direction. On the other hand, if $\bar{Q}\left(\mathcal{C}_{v}^{+}\right)$is negative, 
the flux $\Delta J\left(\mathcal{C}_{v}^{+}\right)$is negative as well. Then the system evolves on the cycle $\mathcal{C}_{v}$ in the minus direction and dissipates the heat $\bar{Q}\left(\mathcal{C}_{v}^{-}\right)=-\bar{Q}\left(\mathcal{C}_{v}^{+}\right)$. Thus, the flux on the cycle $\mathcal{C}_{v}$ appears always in the direction, in which the corresponding heat release $\bar{Q}\left(\mathcal{C}_{v}^{+}\right)$or $\bar{Q}\left(\mathcal{C}_{v}^{-}\right)$, is positive. In this sense, (8) incorporates the second law of thermodynamics on the level of motor cycles.

\section{Operation Mode Diagram}

For a molecular motor with constant step length $\ell$, the velocity $v$ is in general given by

$$
v / \ell=\sum_{v} m_{v}^{+} \Delta J\left(\mathcal{C}_{\nu}^{+}\right)=\sum_{v} m_{\nu}^{+} J\left(\mathcal{C}_{v}^{+}\right)\left(1-e^{-n_{v}^{+} \Delta \bar{\mu}+m_{v}^{+} \bar{F}}\right)
$$

Here (3) and (8) have been used. In the same way in which the motor velocity describes the mechanical performance of a molecular motor, the ATP hydrolysis rate $h$ characterizes the motors chemical performance. The latter rate is given by

$$
h=\sum_{v} n_{v}^{+} \Delta J\left(\mathcal{C}_{v}^{+}\right)=\sum_{v} n_{\nu}^{+} J\left(\mathcal{C}_{\nu}^{+}\right)\left(1-e^{-n_{v}^{+} \Delta \bar{\mu}+m_{v}^{+} \bar{F}}\right) .
$$

The condition $v\left(\bar{F}_{\mathrm{s}}\right)=0$ defines the stall force $\bar{F}_{\mathrm{s}}$, which can be calculated via (9). Equivalently, one can define a balancing chemical potential $\Delta \bar{\mu}_{\mathrm{b}}$ for the zeros of the hydrolysis rate $h\left(\Delta \bar{\mu}_{\mathrm{b}}\right)=0$ via (10).

In a network representation with only ATP binding triggered stepping, $\langle 25\rangle$ in Fig. 2, and a negligible P release triggered stepping transition, $\langle 14\rangle$ in Fig. 2, both mechanical and chemical slip cycles where found to be negligible as well. For this tightly coupled system explicit relations for the stall force as a function of the chemical potential difference $\Delta \bar{\mu}$ and the balancing chemical potential as a function of the load force $\bar{F}$ have been presented in $[29,32]$, see Fig. 3.

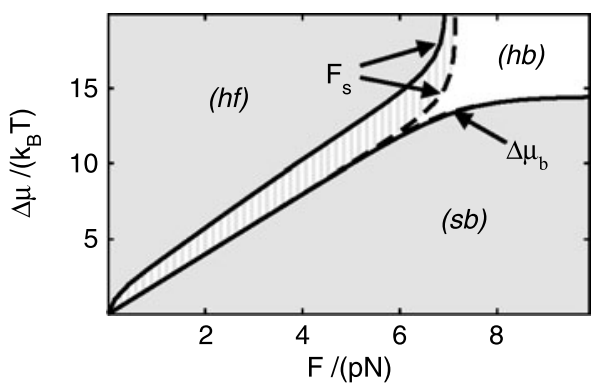

Fig. 3 Operation diagram depending on chemical potential difference $\Delta \mu$ and load force $F$. The stall force $F_{\mathrm{S}}=F_{\mathrm{S}}(\Delta \mu)$ and the balancing chemical potential $\Delta \mu_{\mathrm{b}}=\Delta \mu_{\mathrm{b}}(F)$ are defined via $v\left(F_{\mathrm{S}}\right)=0$ and $h\left(\Delta \mu_{\mathrm{b}}\right)=0$, respectively. The $(h f)$ region corresponds to ATP hydrolysis coupled to forward stepping, the $(s b)$ region to ATP synthesis coupled to backward stepping, and the $(h b)$ region to ATP hydrolysis coupled to backward stepping. Dashed lines are for the tightly coupled model studied in [29]. Solid lines are for the loosely coupled model with transition rates as in Table 1 . The latter model involves significant contributions from a mechanical slip cycle. As a result the $(h b)$ region exhibits a substantial width even close to the origin, which separates the $(h f)$ and the $(s b)$ regions 


\subsection{Linear Response Near Chemomechanical Equilibrium}

Here we want to focus on the linear response regime. Using a series expansion of (9) and (10) the motor velocity $v$ and the ATP hydrolysis rate $h$ are given by

$$
v / \ell \approx-\left(\sum_{v} m_{v}^{+2} J_{0}\left(\mathcal{C}_{v}^{+}\right)\right) \bar{F}+\left(\sum_{v} m_{v}^{+} n_{v}^{+} J_{0}\left(\mathcal{C}_{v}^{+}\right)\right) \Delta \bar{\mu}
$$

and

$$
h \approx-\left(\sum_{\nu} m_{\nu}^{+} n_{v}^{+} J_{0}\left(\mathcal{C}_{v}^{+}\right)\right) \bar{F}+\left(\sum_{\nu} n_{v}^{+2} J_{0}\left(\mathcal{C}_{v}^{+}\right)\right) \Delta \bar{\mu}
$$

to leading order, where $\left.J_{0}\left(\mathcal{C}_{\nu}^{+}\right) \equiv J\left(\mathcal{C}_{v}^{+}\right)\right|_{\bar{F}=0, \Delta \bar{\mu}=0}$. Note that (11) and (12) satisfy Onsager's reciprocity relations as expected [4]. In general the dicycle fluxes $J\left(\mathcal{C}_{v}^{+}\right)$depend on the load force $F$ and on the concentrations [ATP], [ADP] and [P], separately. Since $[\mathrm{ATP}]=[\mathrm{ADP}][\mathrm{P}] \exp (\Delta \bar{\mu}) / K^{\mathrm{eq}}$, they can be written as functions of the load force $F$, the chemical potential $\Delta \mu$, and the product concentrations [ADP] and [P]. Consequently, the dicycle fluxes at chemomechanical equilibrium $J_{0}\left(\mathcal{C}_{v}^{+}\right)$remain functions of the product concentrations [ADP] and [P]. The dicycle excess fluxes $\Delta J\left(\mathcal{C}_{v}^{+}\right)$vanish at equilibrium, because the corresponding dissipated heat is zero $Q\left(\mathcal{C}_{v}^{+}\right)=0(3)$, which leads to $J_{0}\left(\mathcal{C}_{v}^{-}\right)=$ $J_{0}\left(\mathcal{C}_{v}^{+}\right)(8)$.

Equations (11) and (12) lead to the following functions of the stall force and the balancing chemical potential, near the equilibrium $(\bar{F}, \Delta \bar{\mu})=(0,0)$,

$$
\bar{F}_{\mathrm{s}}(\Delta \bar{\mu})=\frac{\sum_{v} m_{v}^{+} n_{v}^{+} J_{0}\left(\mathcal{C}_{v}^{+}\right)}{\sum_{\nu} m_{v}^{+2} J_{0}\left(\mathcal{C}_{v}^{+}\right)} \Delta \bar{\mu}=\zeta_{1} \Delta \bar{\mu}
$$

and

$$
\Delta \bar{\mu}_{\mathrm{b}}(\bar{F})=\frac{\sum_{v} m_{\nu}^{+} n_{v}^{+} J_{0}\left(\mathcal{C}_{v}^{+}\right)}{\sum_{\nu} n_{v}^{+2} J_{0}\left(\mathcal{C}_{v}^{+}\right)} \bar{F}=\zeta_{2} \bar{F} .
$$

From these results one finds $0 \leq \zeta_{1} \zeta_{2} \leq 1$. The sum in the numerator of (13) and (14) includes only fluxes of chemomechanical cycles and no slip cycle fluxes, because for the latter one finds either $m_{v}^{+}=0$ or $n_{v}^{+}=0$. On the other hand, the denominator in (13) includes fluxes of all chemomechanical cycles and all mechanical slip cycles, while the denominator in (14) includes fluxes of all chemomechanical cycles and all chemical slip cycles. Consequently, the coupling ratio $\zeta_{1} \zeta_{2}$ is identical to one for models that exhibit tight coupling between stepping and ATP hydrolysis close to equilibrium. As the fluxes of chemical or mechanical slip cycles increase, the coupling ratio $\zeta_{1} \zeta_{2}$ decreases.

In [29] it has been shown that $-|\Delta \bar{\mu}| \leq \bar{F}_{\mathrm{s}}(\Delta \bar{\mu}) \leq|\Delta \bar{\mu}|$, and $-|\bar{F}| \leq \Delta \bar{\mu}_{\mathrm{b}}(\bar{F}) \leq|\bar{F}|$, i.e. the stall force $\bar{F}_{\mathrm{s}}$ and the balancing chemical potential difference $\Delta \bar{\mu}_{\mathrm{b}}$ lie in the upper and lower, and in the left and right quadrant of the $(\bar{F}-\Delta \bar{\mu})$-plane, respectively. Additionally, the relations (13) and (14) quantify the particular influences of chemomechanical forward stepping, chemomechanical backward stepping, mechanical slip and enzymatic slip cycles on the operation mode diagram near equilibrium.

For the network in Fig. 2, the stall force and the balancing chemical potential in the linear response regime, (13) and (14), are governed by

$$
\zeta_{1}=\frac{J_{0}\left(\mathcal{F}^{+}\right)-J_{0}\left(\mathcal{B}^{+}\right)}{J_{0}\left(\mathcal{F}^{+}\right)+J_{0}\left(\mathcal{B}^{+}\right)+4 J_{0}\left(\mathcal{M}^{+}\right)},
$$


and

$$
\zeta_{2}=\frac{J_{0}\left(\mathcal{F}^{+}\right)-J_{0}\left(\mathcal{B}^{+}\right)}{J_{0}\left(\mathcal{F}^{+}\right)+J_{0}\left(\mathcal{B}^{+}\right)+4 J_{0}\left(\mathcal{E}^{+}\right)}
$$

where $J_{0}\left(\mathcal{F}^{+}\right)=\sum_{\gamma} J_{0}\left(\mathcal{F}_{\gamma}^{+}\right), J_{0}\left(\mathcal{B}^{+}\right)=\sum_{\gamma} J_{0}\left(\mathcal{B}_{\gamma}^{+}\right), J_{0}\left(\mathcal{M}^{+}\right)=\sum_{\gamma} J_{0}\left(\mathcal{M}_{\gamma}^{+}\right)$and $J_{0}\left(\mathcal{E}^{+}\right)=$ $\sum_{\gamma} J_{0}\left(\mathcal{E}_{\gamma}^{+}\right)$, see Appendix A for the list of cycles. Inspection of Fig. 3 shows that $\zeta_{1}<1$ and $\zeta_{2} \simeq 1$. Using these relations in (15) and (16), one can directly conclude that the relevant pathways close to chemomechanical equilibrium are the chemomechanical forward stepping cycles and the mechanical slip cycles. First, it follows from (16), with $\zeta_{2} \simeq 1$ that $J_{0}\left(\mathcal{E}^{+}\right) \ll J_{0}\left(\mathcal{F}^{+}\right)$and $J_{0}\left(\mathcal{B}^{+}\right) \ll J_{0}\left(\mathcal{F}^{+}\right)$. Second, using the latter inequality in (15) together with $\zeta_{1}<1$, one concludes that $J_{0}\left(\mathcal{M}^{+}\right)$must be comparable to or even larger than $J_{0}\left(\mathcal{F}^{+}\right)$. In this way, one finds from $\zeta_{1}<1$ and $\zeta_{2} \simeq 1$ together with the relations (15) and (16), that the only relevant fluxes are $J_{0}\left(\mathcal{F}^{+}\right)$and $J_{0}\left(\mathcal{M}^{+}\right)$. These results also explain the different operation mode diagrams as obtained in our previous study [29] that ignored mechanical slip cycles, and in another study [34] that is based on ratchet systems and implicitly incorporates mechanical slips.

As illustrated in Fig. 3, the slower increase of the stall force for a system with mechanical slip leads to a gap between the operation modes of ATP hydrolysis driven forward stepping $(h f)$ and mechanical force driven ATP synthesis $(s b)$. It is interesting that this gap can be explained by linear response considerations although it extends up to a region far from equilibrium.

\subsection{Operation Mode Boundaries Far from Chemomechanical Equilibrium}

Far from chemomechanical equilibrium one deals with four different operation mode boundaries. The stall force for large chemical driving due to ATP hydrolysis $F_{\mathrm{s}}(\Delta \mu \rightarrow \infty)$, the balancing chemical potential difference for large resisting forces $\Delta \mu_{\mathrm{b}}(F \rightarrow \infty)$, both shown in Fig. 3 for a specific system, the stall force for large chemical driving due to ATP synthesis $F_{\mathrm{s}}(\Delta \mu \rightarrow-\infty)$, and finally the balancing chemical potential difference for large assisting forces $\Delta \mu_{\mathrm{b}}(F \rightarrow-\infty)[29]$.

Each of these operation mode boundaries far from chemomechanical equilibrium arises from the competition between the dominant motor cycles corresponding to the four operation modes. E.g. for large $\Delta \bar{\mu}$, the motor velocity (9) is found to have the form

$$
v / \ell \approx \sum_{\gamma=1}^{N_{\mathcal{F}}} m_{\gamma}^{+} J\left(\mathcal{F}_{\gamma}^{+}\right)+\sum_{\gamma=1}^{N_{\mathcal{B}}} m_{\gamma}^{+} J\left(\mathcal{B}_{\gamma}^{+}\right)+\sum_{\gamma=1}^{N_{\mathcal{M}}} m_{\gamma}^{+} J\left(\mathcal{M}_{\gamma}^{+}\right)\left(1-e^{m_{\gamma}^{+} \bar{F}}\right) .
$$

It is evident from (17) that a finite stall force $v\left(F_{\mathrm{s}}\right)=0$ can only be found in the far from chemical equilibrium regime for network representations that include in addition to chemomechanical forward stepping cycles, chemomechanical backward stepping cycles and/or mechanical slip cycles.

\section{Motor Efficiency}

For a molecular motor, that moves with velocity $v$ against a load force $F$ powered by ATP hydrolysis with rate $h$ the efficiency has the form

$$
\eta=\frac{F v}{\Delta \mu h}=\frac{\bar{F}(v / \ell)}{\Delta \bar{\mu} h}, \quad \text { for } 0 \leq F \leq F_{\mathrm{s}}, \Delta \mu \geq 0,
$$



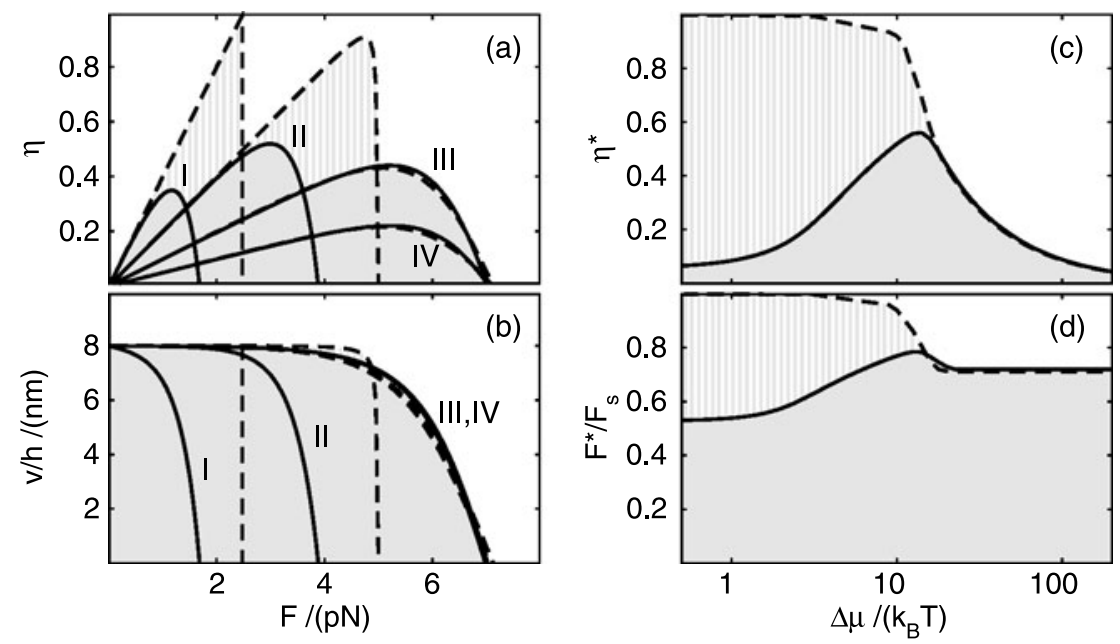

Fig. 4 (a) Motor efficiency $\eta$ as a function of load force $F$ for different values of the chemical potential difference $\Delta \mu$ increasing from left to right: (I) $\Delta \mu=5 k_{B} T$, (II) $10 k_{B} T$, (III) $20 k_{B} T$ and (IV) $40 k_{B} T$. The efficiency $\eta$ vanishes for $F=0$ and $F=F_{\mathrm{S}}$, and exhibits a maximum at $F=F^{*}$ with $0<F^{*}<F_{\mathrm{S}}$. (b) Ratio of motor velocity $v$ to ATP hydrolysis rate $h$, corresponding to the same values of $\Delta \mu$ as in (a). The plots for (III) $\Delta \mu=20 k_{B} T$ and (IV) $\Delta \mu=40 k_{B} T$ are indistinguishable, indicating a saturation of both motor velocity $v$ and ATP hydrolysis rate $h$. Below the stall force, this ratio gives the average distance over which the motor moves per hydrolyzed ATP molecule. (c) Maximal efficiency $\eta^{*}=\eta\left(F^{*}\right)$ as a function of the chemical potential difference $\Delta \mu$, see (18). (d) Fraction of load of maximal efficiency $F^{*}$ and stall force $F_{\mathrm{s}}$. In all figures, dashed lines correspond to the tightly coupled model and solid lines to the loosely coupled model, see also Fig. 3

where the chemical potential difference $\Delta \bar{\mu}$ has been defined in (4). Note that the stall force $F_{\mathrm{s}}$ depends on the ATP, ADP and P concentrations, and consequently on $\Delta \mu$.

Figure 4(a) shows the load dependence of the efficiency explicitly for different chemical potential differences. For $F=0$ and $F=F_{\mathrm{s}}$ the efficiency vanishes. In between it attains a maximal value at $F^{*}$. The efficiency as defined in (18) is the ratio of the load force $F$ and the chemical potential difference $\Delta \mu$ times the step length per hydrolyzed ATP molecule, which is given by $v / h$. The latter value is plotted in Fig. 4(b) and illustrates the loss of the tight coupling with increasing loads. For small loads the kinesin motor needs exactly one ATP molecule per step, which is consistent with experimental observations, [19]. With increasing loads, however, the distance per ATP molecule decreases and reaches zero at stall force $F_{\mathrm{s}}$. In other words, the number of ATP molecules per step increases with increasing loads. As one moves further away from chemical equilibrium by increasing the chemical potential difference, not only the stall force increases but also the load regime of tight coupling, compare the plateau in Fig. 4(b), for which the ratio $v / h$ is essentially constant and close to $v / h=8 \mathrm{~nm}$. Far away from equilibrium with $\Delta \mu \gtrsim 20 k_{B} T$, the form of $v / h$ as a function of $F$ becomes independent of $\Delta \mu$, and assumes a certain limiting form.

The maximal efficiency $\eta^{*}=\eta\left(F^{*}\right)$ as a function of the chemical potential difference, Fig. 4(c), exhibits three regimes. Close to chemical equilibrium, the maximal efficiency is independent of $\Delta \mu$. Far from equilibrium the maximal efficiency decreases with $1 / \Delta \mu$. In between these two cases one finds an intermediate regime in which $\eta^{*}$ may reach a maximum. 


\subsection{Motor Efficiency Close to Chemomechanical Equilibrium}

Using (11) and (12) for the motor velocity and the ATP hydrolysis rate in the linear response regime, the efficiency (18) becomes

$$
\eta \approx \frac{-\bar{F}^{2} / \zeta_{1}+\Delta \bar{\mu} \bar{F}}{\Delta \bar{\mu}^{2} / \zeta_{2}-\Delta \bar{\mu} \bar{F}},
$$

for small values of $\bar{F}$ and $\Delta \bar{\mu}$. It then follows from $\partial \eta / \partial F=0$ that the load of maximal efficiency increases linearly with the chemical potential difference as $F^{*} \approx\left(1 / \zeta_{2}\right)(1-$ $\left.\sqrt{1-\zeta_{1} \zeta_{2}}\right) \Delta \bar{\mu}$, and that the maximal efficiency near equilibrium becomes independent of $\Delta \bar{\mu}$, see Fig. 4(c),

$$
\eta^{*}=\eta\left(F^{*}\right) \approx \frac{2}{\zeta_{1} \zeta_{2}}\left(1-\sqrt{1-\zeta_{1} \zeta_{2}}\right)-1
$$

It is interesting to note that a relation similar to (20) has been previously obtained by Hill for a filament sliding model of filament contraction, see (169) in [35], as well as in Refs. [4, 36], where the product $\zeta_{1} \zeta_{2}$ was denoted by $\Lambda$. However, as far as we know, our relations (13) and (14) that express the coupling constants $\zeta_{1}$ and $\zeta_{2}$ in terms of the dicycle fluxes $J_{0}\left(\mathcal{C}_{v}^{+}\right)$ have not been derived before. The relation (20) has the asymptotic behavior $\eta^{*} \approx 1$ for tight coupling with $\zeta_{1} \zeta_{2}=1$, and $\eta^{*} \approx \zeta_{1} \zeta_{2} / 4$ for loose coupling, i.e. $\zeta_{1} \zeta_{2} \ll 1$, which arises from the appearance of chemical or mechanical slip cycles.

Thus, the degree of coupling between ATP hydrolysis and stepping at chemomechanical equilibrium determines also the intermediate regime of the efficiency, see Fig. 4(c). For sufficiently tight coupling, $\eta^{*}$ reaches its maximum for small $\Delta \mu$. However for loose coupling at chemomechanical equilibrium $\eta^{*}$ increases with increasing the chemical potential difference and reaches its maximal value at finite $\Delta \mu$.

\subsection{Motor Efficiency Far from Chemomechanical Equilibrium}

A motor molecule is driven far from chemical equilibrium as long as the substrate concentrations are much larger than the product concentrations. Commonly, single molecule assays of processively walking molecular motors are performed at negligible small ADP and P concentrations. In this situation the motor is driven far from chemical equilibrium, since $\Delta \bar{\mu}$ as given in (4) is relatively large, even for small (rate limiting) ATP concentrations.

In the limit of large $\Delta \mu$, the dicycle fluxes remain functions of the load force and the product concentrations. Hence, far from chemical equilibrium, the stall force, the force of maximal efficiency and the maximal efficiency itself depend on the product concentrations. However, for the network representation of the kinesin motor in Fig. 2 this dependence turns out to be relatively weak, [29].

Since far from chemical equilibrium, with large $\Delta \mu$, both the motor velocity and the ATP hydrolysis rate become independent of the chemical potential difference $\Delta \mu$, the efficiency as given by (18) decreases as $\eta \sim 1 / \Delta \mu$. This non-equilibrium regime is characterized by a constant stall force $F_{\mathrm{s}}$ and constant force of maximal efficiency $F^{*}$, Fig. 4(d).

\section{Summary}

The energy transduction of molecular motors is determined by the interplay between alternative and competitive motor cycles. In addition to chemomechanical cycles that couple 
energetically favorable processes of fuel consumption to the performance of useful work, chemical or mechanical slip cycles also play a role in the motor kinetics [12, 32]. In this article, we studied the relation between the fluxes on different motor cycles and the dynamics of the kinesin motor. For given thermodynamic conditions, as provided by the substrate concentration [ATP], the product concentrations [ADP] and [P], and the load force $F$, the kinesin motor hydrolyzes ATP with a constant rate $h$ and moves with constant velocity $v$.

For a large chemical potential difference $\Delta \mu$ for ATP hydrolysis and small load force $F$, the hydrolysis of ATP drives the kinesin motor to step forward against the load force, see mode $(h f)$ in Fig. 3. For small $\Delta \mu$ and large load forces, the kinesin motor is pulled backwards to induce the synthesis of ATP, see mode $(s b)$ in Fig. 3. Finally for large $\Delta \mu$ and large $F$ corresponding to mode $(h b)$ in Fig. 3, the ATP hydrolysis driven by a large value of $\Delta \mu$ and the backward stepping induced by a large load force $F$ may be regarded as two uncoupled processes. The operation mode boundaries are given by the stall force $F_{\mathrm{s}}$ and the balancing chemical potential difference $\Delta \mu_{\mathrm{b}}$, which are functions of the chemical potential difference $\Delta \mu$ and the load force $F$, respectively.

The stall force increases linearly with $\Delta \mu$ for small $\Delta \mu$ but saturates for large $\Delta \mu$. We showed that the slope of the linear increase can be expressed in terms of the different dicycle fluxes at equilibrium, see (13) and (15): This slope depends on the fluxes of the chemomechanical cycles and mechanical slip cycles but is independent of the fluxes on chemical or enzymatic slip cycles. It turns out, that the increase of the stall force is $F_{\mathrm{s}}=\Delta \mu / \ell$ for tightly coupled models, i.e. when the fluxes of the mechanical slip cycles at equilibrium are negligible. On the other hand, models that exhibit a relevant flux on a mechanical slip cycle at chemomechanical equilibrium lead to a smaller increase of the stall force with $\Delta \mu$. As a result one finds a gap between the mode of ATP hydrolysis driven forward stepping and the mode of backward stepping driven ATP synthesis, see Fig. 3. Since the linear response regime is limited to $\Delta \mu \lesssim k_{B} T$ (see Fig. 4(c,d)) it is surprising that this gap, which is a prominent feature of the whole operation mode diagram, can be explained by linear response analysis. For the motors considered here, the gap, that opens up in the linear response regime, persists into the nonlinear regime, see Fig. 3. It remains to be seen if this is a universal feature that applies to other motors as well.

The linear response regime of the operation mode boundaries and the motor efficiency have also been discussed in $[4,36]$ in the context of ratchet models, but these models did not incorporate the different nucleotide states of the dimeric motors and, thus, cannot distinguish between the different motor cycles. In contrast, our approach has the advantage of directly characterizing the different motor cycles and their free energy transduction ability. Furthermore, this network representation of the motor dynamics allowed us to relate the linear response coefficients with the fluxes on different classes of motor cycles, see (11) and (12).

Since the investigation of the motor dynamics close to chemical equilibrium requires very small substrate concentrations and consequently leads to very small turnover rates, experimental studies of the operation mode boundaries are difficult in this regime. For the kinetic model studied here, the motor velocity is about $0.5 \mathrm{~nm} / \mathrm{s}$ for $[\mathrm{ADP}]=[\mathrm{P}]=10 \mathrm{mM}$, [ATP] $=5 \mu \mathrm{M}$, corresponding to $\Delta \mu=10 k_{B} T$, and $F=0$. Such a small velocity can hardly be determined in single molecule experiments. However, in cooperative transport by several motors [37], a significant increase of the observation time can be achieved, which might also be used in future studies to determine the operation modes close to equilibrium. One should then be able to distinguish motors with and without mechanical slip cycles: in the absence of such a cycle, the stall force $F_{\mathrm{s}}$ would be equal to $\Delta \mu / \ell$ for small $\Delta \mu$, whereas this force would be significantly smaller than $\Delta \mu / \ell$ in the presence of a mechanical slip cycle, see Fig. 3. 
The chemomechanical coupling not only determines the different operation modes of the molecular motor, but also the efficiency of the corresponding energy transduction processes. In the present article we focused on the efficiency of ATP hydrolysis driven forward stepping, since this is the only regime which has been systematically studied so far.

For the kinesin network representation one finds a linear increase for the efficiency $\eta$ with increasing load forces $F$ for small $F$ and constant $\Delta \mu$. The efficiency increases in this regime simply because the supplied chemical energy can be used to perform mechanical work and need not be wasted in terms of heat. A further increase of the load force leads to an increase of the fluxes on chemical and mechanical slip cycles as well. Consequently the coupling between ATP hydrolysis and stepping becomes looser, and the efficiency of energy transduction decreases, see Fig. 4 . Thus, between $F=0$ and $F=F_{\mathrm{s}}$ the efficiency attains the maximum value $\eta=\eta^{*}$ at $F=F^{*}$.

In the limit of negligible chemical driving corresponding to small $\Delta \mu$, the maximal efficiency $\eta^{*}$ reaches a value that depends on the relevance of the chemical and mechanical slip cycles at equilibrium. For tight coupling, i.e., when the fluxes of the slip cycles are negligible, $\eta^{*}$ reaches its maximum of $\eta^{*}=1$ close to chemomechanical equilibrium with $\Delta \mu=0$, see (20). On the other hand, if chemical and/or mechanical slip cycles have larger fluxes than the chemomechanical cycles, one finds $\eta^{*} \ll 1$, as shown by the solid lines in Fig. 4. The latter situation corresponds to loose chemomechanical coupling.

For large $\Delta \mu$, both the motor velocity $v$ and the ATP hydrolysis rate $h$ saturate. For kinesin, such a saturation is attained for $\Delta \mu>10 k_{B} T$ as shown in Fig. 4. From the definition (18) of the efficiency, it then follows that $\eta^{*}$ decreases with increasing chemical potential difference as $1 / \Delta \mu$. This behavior is universal and does not depend on the absence or presence of slip cycles.

Open Access This article is distributed under the terms of the Creative Commons Attribution Noncommercial License which permits any noncommercial use, distribution, and reproduction in any medium, provided the original author(s) and source are credited.

\section{Appendix A: Catalytic Cycles of the Reduced Network Representation}

The reduced network representation for the processive kinesin walk in Fig. 2 includes 12 different catalytic cycles, which are listed in the following.

(i) chemomechanical forward stepping cycles:

$$
\begin{aligned}
& \mathcal{F}_{1}=\langle 12561\rangle, \\
& \mathcal{F}_{2}=\langle 14561\rangle, \\
& \mathcal{F}_{3}=\langle 12571\rangle, \\
& \mathcal{F}_{4}=\langle 14571\rangle ;
\end{aligned}
$$

(ii) chemomechanical backward stepping cycles:

$$
\begin{aligned}
& \mathcal{B}_{1}=\langle 45234\rangle, \\
& \mathcal{B}_{2}=\langle 41234\rangle ;
\end{aligned}
$$


(iii) mechanical slip cycles:

$$
\begin{aligned}
& \mathcal{M}_{1}=\langle 1432561\rangle, \\
& \mathcal{M}_{2}=\langle 1432571\rangle ;
\end{aligned}
$$

(iv) enzymatic slip cycles:

$$
\begin{aligned}
& \mathcal{E}_{1}=\langle 1234561\rangle, \\
& \mathcal{E}_{2}=\langle 1234571\rangle .
\end{aligned}
$$

In addition one finds the two thermal slip cycles $|14521\rangle$ and $|16571\rangle$ that do not contribute to energy transduction and carry no net flux.

\section{Appendix B: Rate Constants}

The dynamics of the kinetic network representation is assumed to be Markovian, with transition rates

$$
\omega_{i j}=\kappa_{i j} \Phi_{i j}\left[\mathrm{X}_{i j}\right]
$$

Table 1 Rate constants, (25) of the network model in Fig. 1 to reproduce the observed motor properties of $[17,26]$. Values of ATP binding and release, ADP release and P release are consistent with experimental knowledge, see e.g. [38] for review

\begin{tabular}{lll}
\hline & $|i j\rangle$ & \\
\hline$(\mathrm{D}, \mathrm{E}) \rightarrow(\mathrm{E}, \mathrm{D})$ & $|14\rangle$ & $\kappa_{i j} / \mathrm{s}$ \\
$(\mathrm{E}, \mathrm{D}) \rightarrow(\mathrm{D}, \mathrm{E})$ & $|41\rangle$ & $0.01^{\star}$ \\
$(\mathrm{D}, \mathrm{T}) \rightarrow(\mathrm{T}, \mathrm{D})$ & $|25\rangle$ & $100^{\star}$ \\
$(\mathrm{T}, \mathrm{D}) \rightarrow(\mathrm{D}, \mathrm{T})$ & $|52\rangle$ & $4.1 \times 10^{4}$ \\
$(\mathrm{D}, \mathrm{E}) \rightarrow(\mathrm{D}, \mathrm{T})$ & $|12\rangle$ & 0.27 \\
$(\mathrm{D}, \mathrm{T}) \rightarrow(\mathrm{D}, \mathrm{E})$ & $|21\rangle$ & $2 / \mu \mathrm{M}$ \\
$(\mathrm{T}, \mathrm{D}) \rightarrow(\mathrm{T}, \mathrm{E})$ & $|56\rangle$ & 100 \\
$(\mathrm{~T}, \mathrm{E}) \rightarrow(\mathrm{T}, \mathrm{D})$ & $|65\rangle$ & 110 \\
$(\mathrm{~T}, \mathrm{E}) \rightarrow(\mathrm{D}, \mathrm{E})$ & $|61\rangle$ & $0.06 / \mu \mathrm{M}$ \\
$(\mathrm{D}, \mathrm{E}) \rightarrow(\mathrm{T}, \mathrm{E})$ & $|16\rangle$ & 110 \\
$(\mathrm{~T}, \mathrm{D}) \rightarrow(\mathrm{D}, \mathrm{D})$ & $|57\rangle$ & $0.01 / \mu \mathrm{M}$ \\
$(\mathrm{D}, \mathrm{D}) \rightarrow(\mathrm{T}, \mathrm{D})$ & $|75\rangle$ & 110 \\
$(\mathrm{D}, \mathrm{D}) \rightarrow(\mathrm{D}, \mathrm{E})$ & $|71\rangle$ & $2 \times 10^{-4} / \mu \mathrm{M}$ \\
$(\mathrm{D}, \mathrm{E}) \rightarrow(\mathrm{D}, \mathrm{D})$ & $|17\rangle$ & 110 \\
$(\mathrm{E}, \mathrm{D}) \rightarrow(\mathrm{T}, \mathrm{D})$ & $|45\rangle$ & $3.1 / \mu \mathrm{M}$ \\
$(\mathrm{T}, \mathrm{D}) \rightarrow(\mathrm{E}, \mathrm{D})$ & $|54\rangle$ & $2 / \mu \mathrm{M}$ \\
$(\mathrm{D}, \mathrm{T}) \rightarrow(\mathrm{E}, \mathrm{T})$ & $|23\rangle$ & $7 \times 10^{-9}$ \\
$(\mathrm{E}, \mathrm{T}) \rightarrow(\mathrm{D}, \mathrm{T})$ & $|32\rangle$ & 110 \\
$(\mathrm{E}, \mathrm{T}) \rightarrow(\mathrm{E}, \mathrm{D})$ & $|34\rangle$ & $4 \times 10^{-4} / \mu \mathrm{M}$ \\
$(\mathrm{E}, \mathrm{D}) \rightarrow(\mathrm{E}, \mathrm{T})$ & $|43\rangle$ & 110 \\
\hline
\end{tabular}


Table 2 Parameters of the load dependent factors of the chemical transition rates, (25) and (26), in the network model in Fig. 1 to reproduce the observed motor properties of [17]. The load distribution factors of the mechanical transition rates are found to be $\theta_{14}=\theta_{25}=0.55$

\begin{tabular}{lll}
\hline & $\langle i j\rangle$ & $\chi_{i j}$ \\
\hline$(\mathrm{D}, \mathrm{E}) \leftrightarrow(\mathrm{D}, \mathrm{T})$ & $\langle 12\rangle$ & 0.4 \\
$(\mathrm{~T}, \mathrm{D}) \leftrightarrow(\mathrm{T}, \mathrm{E})$ & $\langle 56\rangle$ & 0 \\
$(\mathrm{~T}, \mathrm{E}) \leftrightarrow(\mathrm{D}, \mathrm{E})$ & $\langle 61\rangle$ & 0.3 \\
$(\mathrm{~T}, \mathrm{D}) \leftrightarrow(\mathrm{D}, \mathrm{D})$ & $\langle 57\rangle$ & 0.3 \\
$(\mathrm{D}, \mathrm{D}) \leftrightarrow(\mathrm{D}, \mathrm{E})$ & $\langle 71\rangle$ & 0 \\
$(\mathrm{E}, \mathrm{D}) \leftrightarrow(\mathrm{T}, \mathrm{D})$ & $\langle 45\rangle$ & 0.4 \\
$(\mathrm{D}, \mathrm{T}) \leftrightarrow(\mathrm{E}, \mathrm{T})$ & $\langle 23\rangle$ & 0 \\
$(\mathrm{E}, \mathrm{T}) \leftrightarrow(\mathrm{E}, \mathrm{D})$ & $\langle 34\rangle$ & 0.3 \\
\hline
\end{tabular}

that are given by a rate constant $\kappa_{i j}$, a load dependent factor $\Phi_{i j}$, and the activity of species $\mathrm{X}$ that binds during transition $|i j\rangle$.

For the chemical transitions, the load dependent factors are

$$
\Phi_{i j}=\Phi_{j i}=\frac{2}{1+\exp \left(\chi_{i j} \ell F /\left(k_{B} T\right)\right)} .
$$

The mechanical transitions, on the other hand, have load dependencies of $\Phi_{i j}=\exp \left(-\theta_{i j} \ell \times\right.$ $\left.F /\left(k_{B} T\right)\right)$ for the forward stepping direction and $\Phi_{j i}=\exp \left(\left(1-\theta_{i j}\right) \ell F /\left(k_{B} T\right)\right)$ for the corresponding reverse transition.

The rate constants and the load dependencies used to calculate the operation mode diagram and the efficiency are summarized in Tables 1 and 2, respectively.

\section{References}

1. Smoluchowski, M.V.: Experimentell nachweisbare, der üblichen Thermodynamik widersprechende Molekularphänomene. Phys. Z. 13, 1069 (1912)

2. Huxley, A.F.: Muscle structure and theories of contraction. Prog. Biophys. Biophys. Chem. 7, 255 (1957)

3. Peskin, C., Oster, G.: Coordinated hydrolysis explains the mechanical behavior of kinesin. Biophys. J. 68, S202 (1995)

4. Jülicher, F., Ajdari, A., Prost, J.: Modeling molecular motors. Rev. Mod. Phys. 69, 1269 (1997)

5. Reimann, P.: Brownian motors: noisy transport far from equilibrium. Phys. Rep. 361, 57 (2002)

6. Polanyi, M., Wigner, E.: Concerning the interference of natural oscillation as a reason for energy variations and chemical transformation. Z. Phys. Chem. 139, 439 (1928)

7. Eyring, H.: The activated complex in chemical reactions. J. Chem. Phys. 3, 98 (1935)

8. Kramers, H.A.: Brownian motion in a field of force and the diffusion model of chemical reactions. Physica 7, 284 (1940)

9. Haldane, J.B.S.: Enzymes. MIT Press, Cambridge (1965)

10. Hill, T.L.: Interrelations between random walks on diagrams (graphs) with and without cycles. Proc. Natl. Acad. Sci. USA 85, 2879 (1988)

11. Liepelt, S., Lipowsky, R.: Steady-state balance conditions for molecular motor cycles and stochastic nonequilibrium processes. Europhys. Lett. 77, 50002 (2007)

12. Liepelt, S., Lipowsky, R.: Kinesin's network of chemomechanical motor cycles. Phys. Rev. Lett. 98, 258102 (2007)

13. Lipowsky, R., Liepelt, S.: Chemomechanical coupling of molecular motors: thermodynamics, network representations, and balance conditions. J. Stat. Phys. 130, 39 (2008). Erratum, J. Stat. Phys. 135, 777 (2009) 
14. Kolomeisky, A.B., Widom, B.: A simplified "ratchet" model of molecular motors. J. Stat. Phys. 93, 633 (1998)

15. Fisher, M.E., Kolomeisky, A.B.: Simple mechanochemistry described the dynamics of kinesin molecules. Proc. Natl. Acad. Sci. USA 98, 7748 (2001)

16. Fisher, M.E., Kim, Y.C.: Kinesin crouches to sprint but resists pushing. Proc. Natl. Acad. Sci. USA 102, 16209 (2005)

17. Carter, N.J., Cross, R.A.: Mechanics of the kinesin step. Nature 435, 308 (2005)

18. Hill, T.L.: Free Energy Transduction and Biochemical Cycle Kinetics. Springer, New York (1989)

19. Schnitzer, M.J., Block, S.M.: Kinesin hydrolyzes one ATP per 8-nm step. Nature 388, 386 (1997)

20. Hancock, W.O., Howard, J.: Processivity of the motor protein kinesin requires two heads. J. Cell Biol. 140, 1395 (1998)

21. Yildiz, A., Tomishige, M., Vale, R.D., Selvin, P.R.: Kinesin walks hand-over-hand. Science 303, 676 (2004)

22. Uemura, S., et al.: Kinesin-microtubule binding depends on both nucleotide state and loading direction. Proc. Natl. Acad. Sci. USA 99, 5977 (2002)

23. Guydosh, N.R., Block, S.M.: Backsteps induced by nucleotide analogs suggests the front head of kinesin is gated by strain. Proc. Natl. Acad. Sci. USA 103, 8054 (2006)

24. Mori, T., Vale, R.D., Tomishige, M.: How kinesin waits between steps. Nature 450, 750 (2007)

25. Alonso, M.C., Drummond, D.R., Kain, S., Hoeng, J., Amos, L., Cross, R.A.: An ATP gate controls tubulin binding by the tethered head of Kinesin-1. Science 316, 120 (2007)

26. Schief, W.R., Clark, R.H., Crevenna, A.H., Howard, J.: Inhibition of kinesin mobility by ADP and phosphate supports a hand-over-hand mechanism. Proc. Natl. Acad. Sci. USA 101, 1183 (2004)

27. Auerbach, S.D., Johnson, K.A.: Alternating site ATPase pathway of rat conventional kinesin. J. Biol. Chem. 280, 37048 (2005)

28. Taniguchi, Y., Nishiyama, M., Ishii, Y., Yanagida, T.: Entropy rectifies the Brownian steps of kinesin. Nat. Chem. Biol. 1, 342 (2005)

29. Liepelt, S., Lipowsky, R.: Operation modes of the molecular motor kinesin. Phys. Rev. E 79, 011917 (2009)

30. Nishiyama, M., Higuchi, H., Yanagida, T.: Chemomechanical coupling of the forward and backward steps of single kinesin molecules. Nat. Cell Biol. 4, 790 (2002)

31. Hyeon, C., Klumpp, S., Onuchic, J.N.: Kinesin's backsteps under mechanical load. Phys. Chem. Chem. Phys. 11, 4899 (2009)

32. Lipowsky, R., Liepelt, S., Valleriani, A.: Energy conversion by molecular motors coupled to nucleotide hydrolysis. J. Stat. Phys. 135, 951 (2009)

33. Cantelo, R.C.: The 2nd law of thermodynamics in chemistry. J. Phys. Chem. 32, 982-988 (1928)

34. Lacoste, D., Lau, A.W.C., Mallick, K.: Fluctuation theorem and large deviation function for a solvable model of a molecular motor. Phys. Rev. E 78, 011915 (2008)

35. Hill, T.L.: Theoretical formalism for the sliding filament model of contraction of striated muscle, Part I. Prog. Biophys. Mol. Biol. 28, 267 (1974) (1974)

36. Parmeggiani, A., Jülicher, F., Ajdari, A., Prost, J.: Energy transduction of isothermal ratches: Generic aspects and specific examples close to and far away from equilibrium. Phys. Rev. E 60, 2127 (1999)

37. Klumpp, S., Lipowsky, R.: Cooperative cargo transport by several molecular motors. Proc. Natl. Acad. Sci. USA 102, 17284 (2005)

38. Cross, R.A.: The kinetic mechanism of kinesin. Trends Biochem. Sci. 29, 301 (2004) 